\title{
Voltage and Current Sensor for State Estimation in Distribution Network with Generation
}

\author{
C. Gouveia ${ }^{1}$, H. Leite ${ }^{1}$ and I. Ferreira ${ }^{1}$
}

${ }^{1}$ Department of Electrical Engineering FEUP, Porto University

FEUP, Rua Dr. Roberto Frias, s/n 4200-465 Porto PORTUGAL, e-mail: cgouveia@fe.up.pt, hleite@fe.up.pt, imf@fe.up.pt.

\begin{abstract}
The penetration of Distributed Generation into the distribution networks has been increasing during the last decades. However, the increase of generation brings additional technical challenges for the Distribution Network Operators. Thus, the integration of Distributed Generation along with the improvement of the quality of service is driving Distribution Network Operators to actively control their networks. In order to achieve an effective control of the distribution network it is necessary to estimate the voltage on the network. This paper presents a method to accurately estimate voltage on the MV Network from limited measures of voltage and current. A method to determine the best location to place those voltage and current sensors is also explained
\end{abstract}

\section{Key words}

Voltage and current sensor, measurement placement, state estimation, Distribution network, Distributed Generation

\section{Introduction}

The integration of Distributed Generation (DG) along with the improvement of the quality of service is driving Distribution Network Operators (DNO) to actively control their networks. Active control strategies allow DNOs to improve the capability of monitoring, controlling and economically dispatching the distribution systems, as well as increasing the integration of DG [1]. Therefore, many authors have been proposing new tools and control procedures to transform passive distribution networks into active ones. In [1], a Distribution Management System Controller (DMSC) was proposed. In order to take action accordingly, the DMSC requires a state estimation algorithm, which can provide the network voltage in real time. The implementation of a state estimation method in distribution networks is quite challenging due to the lack of real-time measurements available. The state estimation algorithm has to deal with measurements predominantly off-time estimations of loads named pseudo-measurements. Load estimations are affected with great uncertainty, since it is difficult to estimate low voltage loads.
The state estimation algorithm proposed in [1] is based on weighted least squares (WLS) state estimation. The network model adopted is single-phase on the basis because English Medium Voltage (MV) networks are balanced. In [2], the WLS state estimation algorithm performance was statistically evaluated. It was found to be a suitable solver for the distribution systems state estimation. The WLS state estimation algorithm performance was evaluated with three statistical measures such as bias, consistency and quality. It was tested on the 12-bus and 95-bus UK-GDS network models considering the limited redundancy and the large uncertainty affecting the pseudo-measurements. In all cases, the WLS was unbiased and consistent, presenting good quality of the estimates.

To improve the quality of the WLS state estimation results more real time measurements need to be considered [2]. However, the state estimation algorithm developed in [1] ignores current measurements, which also can be measured at distribution networks with simplicity. Current measurements can improve the redundancy of the measurement set as well as the accuracy of the estimated voltages [3]. Considering those advantages, distribution state estimation methods including current measurements were developed in [4] and [5]. However, those state estimation algorithms were developed mainly for American MV networks, which are unbalanced, so that a three-phase model of the network was required. The three-phase state estimation methods are complex and difficult to implement.

The more measurements placed in the distribution networks the greater is the investments needed. Therefore, a compromise is required amongst accuracy, reliability and cost. In [6] a measurement placement method is proposed, which assures accuracy, reliability, cost and bad data requirements. However, this method leads to a large number of measurements placed and greater investments. To obtain a limited number of measurements, in [1] it was proposed a placement method included in the DMSC, which places voltage measurements in Medium Voltage (MV) to Low Voltage (LV) switching boards. The method started with the existent real measurements and pseudo-measurements 
and then placed voltage measurements in the locations with the larger standard deviation. This recursive algorithm runs until a sufficiently small deviation of the estimated voltages is obtained. As a result, a limited number of measurements is required and a compromise between measurement and investment is obtained.

The installation of new measuring equipment, auxiliary equipment and communication infrastructures represent a large investment. New types of measuring equipment are being developed to reduce this investment [7]. MV voltage and current sensor is non-conventional measuring equipment that is already available in the market. This sensor integrates in the same equipment protection and measurement functions, resulting in a reduction of the number of system components and wiring [7]. Comparing to inductive instrument transformers, the voltage and current sensors are not subject to saturation effect, have the same accuracy and are easier to install [7].

This paper describes an algorithm to place voltage and current sensors for state estimation in the distribution network. The state estimation algorithm developed determines voltage across the MV network and its accuracy. It integrates current measurements from the sensors and DG site. The placement method determines the best location for the sensors, so that the voltages on the MV network are estimated accurately with a reduced investment.

\section{Distribution State Estimation Method}

The distribution state estimation algorithm developed determines the voltage magnitude and its accuracy at all busbars of the MV network. The schematic of Fig. 1 represents the scheme of entry data and output data of the distribution state estimation.

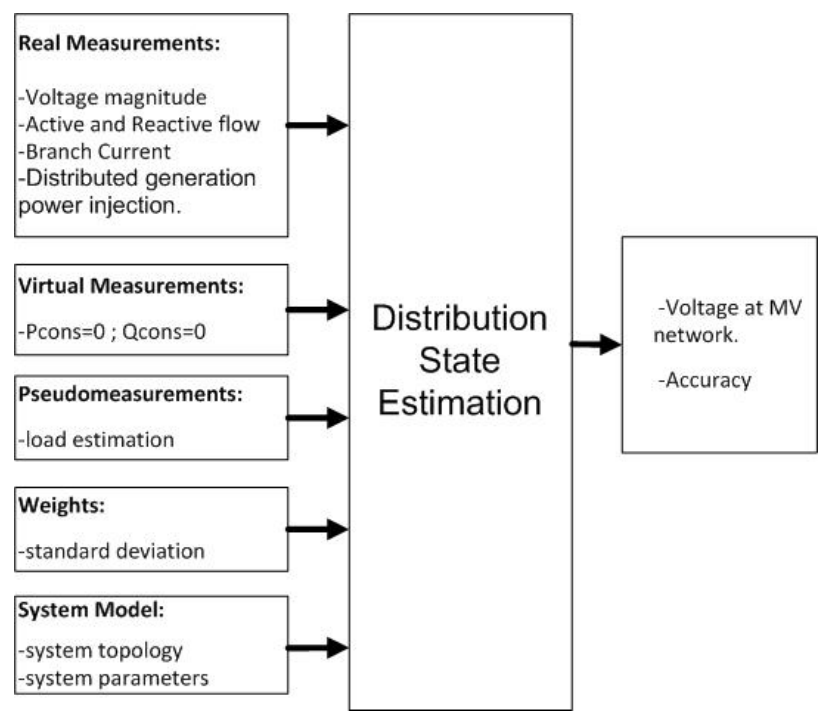

Fig. 1. Scheme of the Distribution State Estimation: entry data and output data.

The state estimation algorithm accommodates a set of real measurements, pseudo-measurements and virtual measurements. The real measurements considered are the voltage, current and power measurements read at the DG site, at the substation MV busbar and from the voltage and current sensors spread across the network. Load estimations at the MV to LV switching boards are modelled as pseudo-measurements. The measurement set is complemented by virtual measurements, which consist of MV busbars where the power injection is null.

The reason behind the system model adopted is singlephase is because the majority of European MV networks are balanced. State estimation credits measurements based on their variance (i.e. given by the squared standard deviation). The standard deviation includes the errors associated with the measurement itself and the communication equipment. It is assumed that measurement errors follow a Gaussian distribution. Therefore, considering a confidence interval of $99.73 \%$, the standard deviation of a measurement is determined using the Eq. 1,

$$
\sigma=z_{i} \times \frac{\operatorname{accuracy}(\%)}{300}
$$

where, $\sigma$ is the standard deviation of the measurement and $z_{i}$ is the measured value.

The distribution state estimation algorithm is based on the weighted least squares approach [8]. The voltage magnitude and angle of all MV busbars (denoted as state variables) are determined by the minimization of the squared error (i.e parameter "e") of all measurements. To find the estimated state of the system it is necessary to minimize the function $\mathrm{J}(\mathrm{x})$ described by Eq. 2,

$$
\min J(x)=\sum_{i=1}^{m} \frac{\left(z_{i}-h_{i}(x)^{2}\right)}{R_{i i}}=[z-h(x)]^{T} R^{-1}[z-h(x)]
$$

where $\mathrm{x}$ is the state variables vector, $\mathrm{m}$ is the number of measurements, $z_{i}$ is the element $i$ of $z$, the vector of the measured values, $h_{i}(x)$ is a nonlinear function relating a measurement to the state variables, $\mathrm{R}_{\mathrm{ii}}$ is the diagonal element from line $\mathrm{i}$ of the weights matrix $\mathrm{R}$. The weights matrix is constituted by measurement variances (i.e. " $\left.\sigma_{\mathrm{m}}^{2} "\right)$.

The minimum of $\mathrm{J}(\mathrm{x})$, see Eq.2, is called the residual vector (i.e. " $\Delta x$ "), given by the Eq. 3,

$$
\Delta x=G^{-1} H^{T} R^{-1}[z-h(x)]
$$

where,

$$
\begin{aligned}
& G=\left[H^{T} R^{-1} H\right] \\
& H(x)=\frac{\partial h(x)}{\partial x}
\end{aligned}
$$

where $\mathrm{G}$ the gain matrix and $\mathrm{H}$ the measurement Jacobian, determined by Eq. 4 and 5 respectively.

The gain matrix $G$ is a matrix significantly sparse and its inversion may cause convergence problems and the deterioration of the state estimation results. Thus, the residual vector shown in Eq. 3 is calculated by the Cholesky decomposition of the gain matrix and using forward/back substitutions [3]. When the system is fully observable the gain matrix can be decomposed into its triangular factors, as in Eq. 6. 


$$
G=L L^{T}
$$

Where $\mathrm{G}$ is the gain matrix and $\mathrm{L}$ is the lower triangular matrix.

The residual vector is determined by solving the Eq. 7, using forward/back substitutions at each iteration $\mathrm{k}$.

$$
L L^{T} \Delta x^{k}=t^{k}
$$

Where,

$$
t^{k}=H^{T} R^{-1}[z-h(x)]
$$

In forward substitution, the aim is to obtain the elements of $\mathrm{u}$, starting from the first element, where $\mathrm{u} 1=\mathrm{t} 1 / \mathrm{L} 11$. After determining $\mathrm{u}$, the residual vector is determined by Eq. 9, starting by the last element of vector $u$.

$$
L^{T} \Delta x^{k}=u
$$

Due to the non-linear nature of $\mathrm{h}(\mathrm{x})$, the solution has to be calculated by an iterative process. The state vector is updated at the end of each iteration $\mathrm{k}$, as stated by Eq. 10 . If the residual vector is higher than the convergence limit, a new measurement jacobian $\mathrm{H}$ and gain matrix $\mathrm{G}$ have to be calculated in order to determine a new residual vector. The iterative process ends when the residual vector is smaller than a predefined convergence threshold. The estimated state of the system is the state vector obtained in the last iteration, (i.e., denoted as $\mathrm{x}^{\mathrm{k}}$ ).

$$
x^{k+1}=x^{k}+\Delta x^{k}
$$

\section{A. Current measurements in state estimation}

Current measurements are available at the main substation, at DG site and at the MV busbars where voltage and current sensors were installed. The state estimation will consider current measurements as they can improve the redundancy of the measurements set and determine the power flow directions, as explained in [3]. In order to eliminate the possibility of multiple solutions and to improve the condition number of the measurement jacobian, squared current magnitude is adopted instead of current magnitude. The deterioration of the measurement Jacobian is due to the strong non-linearity inherent to Eq.11.

$$
I_{i j}=\sqrt{\left(g_{i j}^{2}+b_{i j}{ }^{2}\right)\left(V_{i}^{2}+V_{j}^{2}-2 V_{i} V_{j} \cos \theta_{i j}\right)}
$$

The squared current magnitude is obtained as described by the following Eq. 12,

$$
I_{i j}{ }^{2}=\left(g_{i j}{ }^{2}+b_{i j}{ }^{2}\right)\left(V_{i}^{2}+V_{j}^{2}-2 V_{i} V_{j} \cos \theta_{i j}\right)
$$

where $I_{i j}$ is the branch current magnitude that flows from busbar $\mathrm{i}$ to busbar $\mathrm{j}, \mathrm{g}_{\mathrm{ij}}$ and $\mathrm{b}_{\mathrm{ij}}$ are the branch conductance and susceptance respectively, $V_{i}$ and $V_{j}$ are the voltage magnitudes from busbar $\mathrm{i}$ and $\mathrm{j}$ and $\theta_{\mathrm{ij}}$ is the difference between voltage angle in busbar $\mathrm{i}$ and busbar $\mathrm{j}$.
The squared magnitude current approach has the disadvantage of doubling measurement variances. This way, measurement variances become null for the first iteration being useless to the state estimation. Then, the state variables have to be randomly perturbed for the first iteration.

\section{Measurement Placement Method}

In general MV networks have lack of real measurements. This is actually the primarily challenge for the distribution state estimation algorithm. Therefore, it is essential to place voltage and current measurements at the MV to LV switching boards in order to increase voltage measurement accuracy across the MV network. However, due to cost constraints it is necessary to define carefully the number and the place for those measurements.

The placement method adopted in this paper determines the location for the sensors considering accuracy requirement. The accuracy requirement consists in limiting the uncertainty of the estimated voltages below the uncertainty of voltage magnitude real measurements. This requirement should be respected even in case of communication failure between the control centre and the DG sites. Eq. 13 determines the accuracy of estimated voltages,

$$
\operatorname{accuracy}(\%)=\sqrt{v(x)} \times \frac{300}{x}
$$

where,

$$
v(x)=\operatorname{diag}\left\{G^{-1}\right\}=\operatorname{diag}\left\{\left[H^{T} R^{-1} H\right]^{-1}\right\}
$$

being $x$ the estimated voltage, $v(x)$ its variance, $G$ the gain matrix, $H$ the measurement jacobian and $R$ the weights matrix.

The placement method developed is an offline method and requires real measurements and load estimates to start. If the requirements are violated a voltage and current sensor is placed at the busbar with higher uncertainty. The process is repeated until the requirements are totally fulfilled.

\section{MV Network Case Study}

The distribution network used to validate the measurement placement method and the distribution state estimation algorithm was a 95 busbars network [9]. The network MV voltage is $11 \mathrm{kV}$. This MV network is fed by a single substation with a single on-load tap changing $33 / 11 \mathrm{kV}$. The total number of branches is 94 , being all of them mainly resistive. The average $\mathrm{r}$ to $\mathrm{x}$ ratio of the 94 branches has a relation of 2.12. Between busbar number 87 and 95, there is a normally opened recloser. At the busbars number 11 and 51 there are two wind farms connected. 


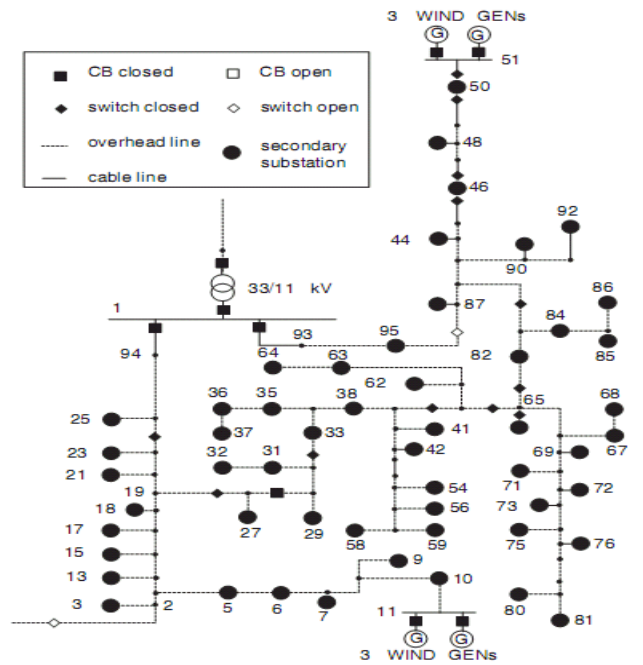

Fig. 2. Distribution Network with 95 busbars

\section{State Estimation Algorithm Placement Method Validation}

and

The state estimation algorithm and the placement method were validated for the following scenarios:

1) Distribution network before reconfiguration (i.e. with the normally open point in its normal state).

2) Inversion of the power flow direction. The wind farms produce the necessary power to feed all the network loads and an excess that goes to the $33 \mathrm{kV}$ network

3) Distribution network re-arranged. The normally open point between busbars 87 and 95 is closed.

For each of those scenarios three cases were considered:

a) full penetration of $\mathrm{DG}$

b) DG disconnected from the network

c) Communication equipment failure at the DG site.

The measurements set is determined by a power flow for each scenario and case and the real measurements are randomly perturbed to simulate a measurement error.

The uncertainty associated to the measurements set was considered as following:

-Voltage magnitude uncertainty: $1 \%$.

- Line current magnitude: $1 \%$.

-Estimated loads uncertainty: 50\%.

-Active line power flow uncertainty: 3\%.

-Generation power injection uncertainty: $3 \%$

To validate the state estimation and the placement method, it was necessary to determine voltage uncertainties before installing any voltage and current sensor. Therefore, cases $\mathrm{a}, \mathrm{b}$ and $\mathrm{c}$ of the three scenarios were simulated, considering only the real measurements available and the load estimates. The results are showed in Fig. 3 to 5.

The limit of acceptable voltage uncertainty considered was $1 \%$. Observing Fig. 3 to 5, the estimated voltage uncertainty level is higher than $1 \%$ for scenarios 1 and 2, when cases a) and c) are considered.

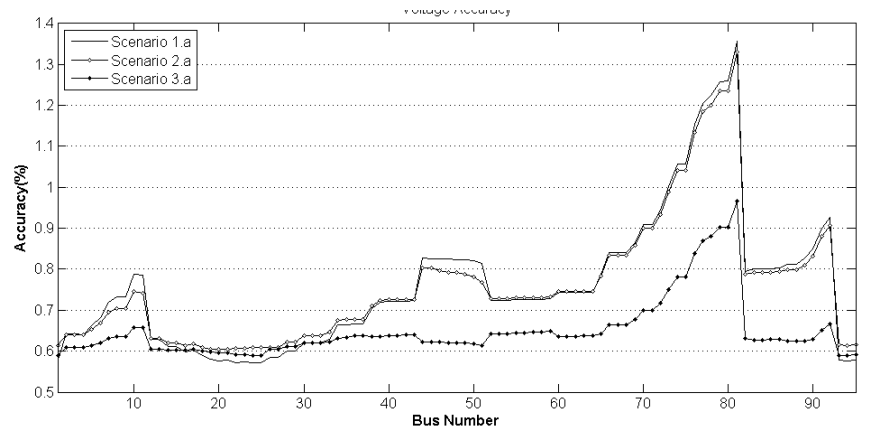

Fig. 3. Comparison of the voltage uncertainty of case a) for scenarios 1), 2) and 3).

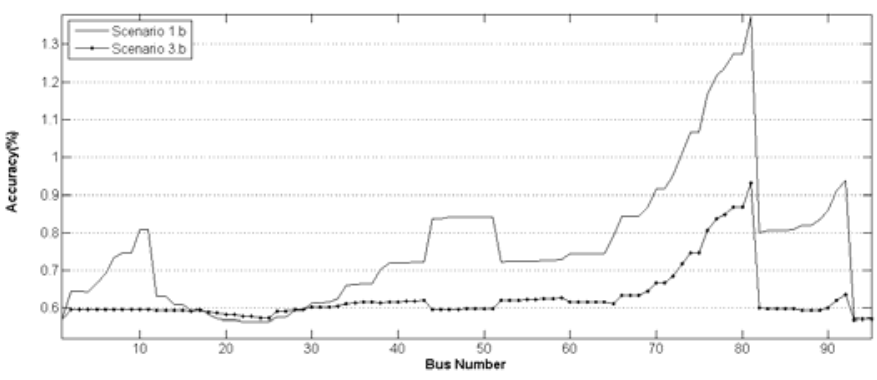

Fig. 4. Comparison of the voltage uncertainty of case b) for scenarios 1), 2) and 3).

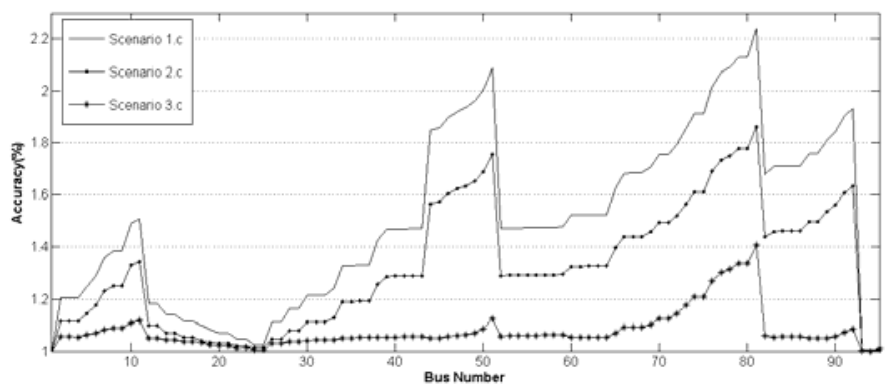

Fig. 5. Comparison of the voltage uncertainty of case c) for the scenarios 1), 2) and 3).

Observing Fig. 3 to 5, the busbar with higher voltage uncertainty is busbar 81 . The load at this busbar is similar to the maximum capacity of the wind farms and the only measurement available is load estimation with an uncertainty of $50 \%$. Therefore, the state estimation does not have enough information to reduce voltage uncertainty at busbar 81 .

Fig. 5 shows that when communication fails with the DG site (case c) ), voltage uncertainty in all busbars increases substantially (above 1\%). Notice that in case c) only the main substation provides real measurements to the state estimator. Therefore, the results show that pseudomeasurements are not sufficient to assure accurate voltage estimations, due to their high uncertainty.

Following the placement method criteria, a voltage and current sensor was placed at the busbar with maximum voltage uncertainty, which was busbar 81 . The results are showed in Fig. 6. 


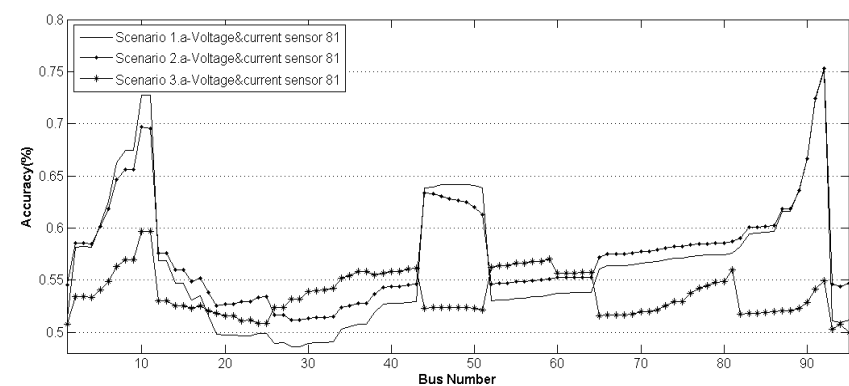

Fig. 6. Comparison of the voltage uncertainty of case a) for the three scenarios 1), 2) and 3), with a voltage and current sensor in busbar 81.

Observing Fig. 6, placing a voltage and current sensor at busbar 81 allowed the state estimator to reduce the voltage uncertainty level below $1 \%$, in cases a) and b). However, observing Fig. 7 , in case of communication failure this solution is not sufficient.

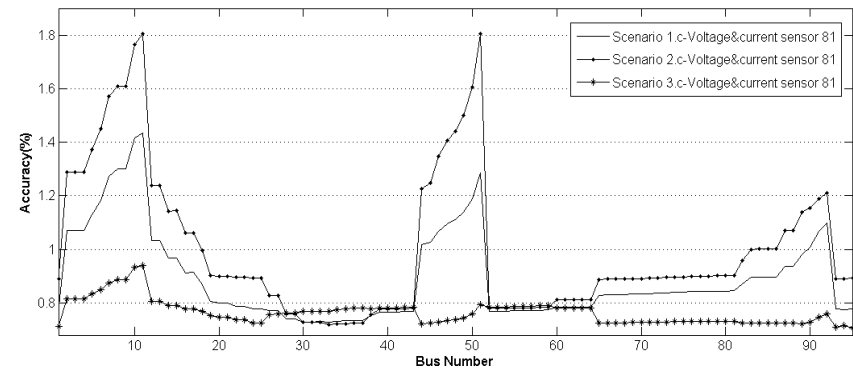

Fig. 7. Comparison of the voltage uncertainty of case c) for the scenarios 1),2) and 3), with a voltage and current sensor in busbar 81 .

Two voltage and current sensors were placed in busbars 10 and 50, so that in case of communication failure, the uncertainty of estimated voltages remains below the defined limit of $1 \%$. Fig. 8 shows that the level of uncertainty dropped below $1 \%$.

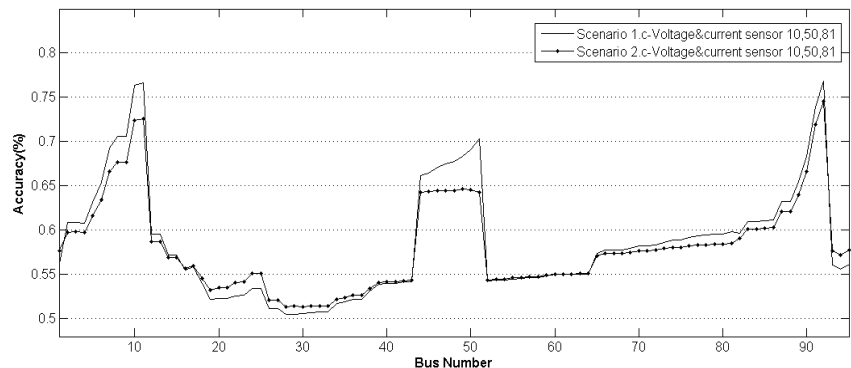

Fig. 8. Comparison of the voltage uncertainty of case c) for the three scenarios 1), 2) and 3), with a voltage and current sensor in busbars 10,50 and 81 .

To obtain accurate voltage magnitude estimation, it was necessary the installation of three voltage and current sensors.

Lastly, it was studied the influence of current measurements on the accuracy of voltage estimation. Thus, the results of adding voltage and current measurements were compared to the results of considering only voltage measurements in busbars 10,50 and 81 .

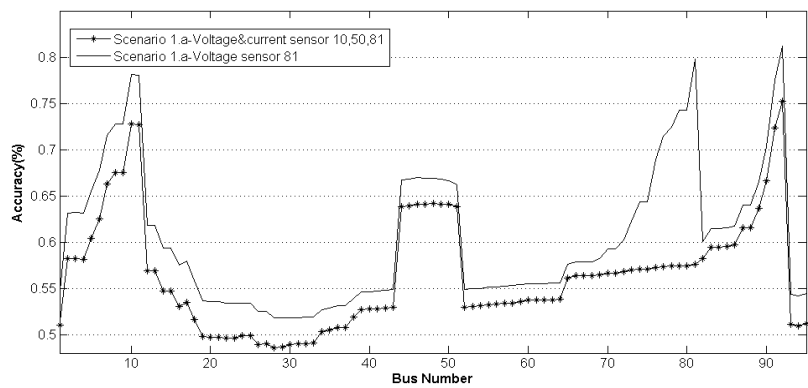

Fig. 9 Comparison of the voltage uncertainty of case a) for the three scenarios 1),2) and 3) with a voltage and current sensor in busbars 10, 50 and 81 .

Observing Fig. 9, estimated voltages are more accurate when using a voltage and current sensor in bus 81 . In fact, not only the voltage uncertainty was reduced at bus 81 , but also in all MV network.

\section{Conclusions}

To continue increasing the amount of generation into the distribution networks, the Distribution Network Operators may need to actively control their networks. In order to actively control the network it is necessary to know the voltage across the network. However, there is lack of real measurements in the network and installing real measurements in all the MV to LV switching boards may be economically impossible.

To assure accurate distribution voltage estimations, this paper presented a state estimation algorithm able to use current measurements as well as a method to place voltage and current sensors along the MV network. The single-phase distribution state estimation algorithm determines the voltage magnitudes across the MV network and their associated uncertainties. To minimize the estimated voltage uncertainty, the placement method determines the minimum number and location of the voltage and current sensors. The results showed that current and voltage measurement together can improve the voltage magnitude accuracy at the MV network.

In summary, the developed method to place voltage and current sensors with the distribution state estimation algorithm may allow distribution network operators to actively control their network and thus increase the amount of generation onto their distribution networks with an improvement in power quality.

\section{Acknowledgement}

This work was financially supported by the "Fundação para a Ciência eTecnologia", (FCT) of Portugal, under the PTDC/EEAENE/73829/2006 project.

\section{References}

[1] Shafiu, A.; Jenkins N.; Strbac, G.; "Measurement location for state estimation of distribution networks with generation", Generation, Transmission and Distribution, IEE Proceedings-Volume 152, Issue 2, 4 March 2005 Page(s):240 - 246. 
[2] Singh, R.; Pal, B.C.; Jabr, R.A.; "Choice of estimator for distribution system state estimation" IET Generation, Transmission \& Distribution, Volume 3, Issue 7, July 2009 Page(s):666 - 678.

[3] Abur ,Ali ; Expósito, Antonio Gómez (2004) "Power system state estimation: theory and implementation", CRC Press, New York.

[4] $\mathrm{Li}, \mathrm{Ke}$ "State Estimation for power distribution system and measurement impacts", IEEE Transactions on Power Systems, Vol. 11, No. 2, Mes 1996, Page(s): 911:916.

[5] Baran, Mesut; Kelley, A.W. "A branch-current-based state estimation method for distribution systems", IEEE Transactions on Power Systems, Volume 10, Issue 1, 1995, Page(s):483 - 491.
[6] Baran, Mesut E.; Zhu, Jinxiang; Zhu, Hongbo; Garren, Kenneth E.; "A meter Placement method for state estimation", IEEE Transactions on Power Systems, Volume 10, no. 3, August 1995, Page(s): 1704-1710.

[7] Spearing, T.; Hauck, K.; Kuchai, M.; "Realising the Benefits of Non-Conventional Sensors", IET 9th International Conference on Developments in Power System Protection on 17-20 March 2008 Page(s):412 - 417.

[8] Schweppe, F.C.; Wildes, J. "Power System Static-State Estimation, Part I: Exact Model", IEEE Transactions on Power Apparatus and Systems, Volume PAS-89, Issue 1, 1970, Page 120 - 125.

[9] Santos, Lúcio (2008), “Aperfeiçoamento de um algoritmo de Estimação de Estados para Rede de Distribuição considerando grande penetração de produção dispersa.", Master thesis, FEUP Porto University, Porto. 